\title{
EVALUATION OF TYPES OF PRE-ANALYTICAL ERRORS IN CLINICAL CHEMISTRY LABORATORY
}

\author{
Amit Ramesh Barapatre1, Avinash Namdeo Jadhao², Manoj Chandrakant Lokhande 3
}

${ }^{1}$ Assistant Professor, Department of Biochemistry, TNMC Nair and B.Y.L. Ch. Hospital.

${ }^{2}$ Assistant Professor, Department of Biochemistry, TNMC Nair and B.Y.L. Ch. Hospital.

${ }^{3}$ Assistant Professor, Department of Biochemistry, TNMC Nair and B.Y.L. Ch. Hospital.

\author{
ABSTRACT \\ OBJECTIVE \\ Evaluation of leading causes of pre-analytical errors in Clinical Biochemistry Laboratory.

\section{MATERIALS AND METHODS} \\ Samples were obtained from the OPD and indoor admitted patients and analysis of the results obtained from clinical chemistry \\ laboratory obtained during one year of study period was done retrospectively. Data was summarised regarding the frequency of \\ factors affecting the pre-analytical quality of results. Laboratory personnel were asked to register all the rejected samples and \\ reasons for rejections.
}

\section{RESULTS}

Out of 62541 inpatient samples collected and screened over period, pre-analytical errors were observed in 4611 samples, which is approximately $3.75 \%$ of the total blood samples received. Insufficient volume of samples accounted for rejection of 2428 samples, which is $1.9 \%$ of total samples received during this period. Haemolysis accounted for rejection of $1.1 \%$ of samples and gross lipaemia was responsible for rejection of $0.4 \%$ samples. $0.26 \%$ of samples were rejected for having wrong/incomplete patient information. Out of 60244 samples collected total from OPD, 5552 samples were rejected for the presence of pre-analytical errors. This accounted for rejection of $4.5 \%$ of total samples received during this period. $2.7 \%$ of samples were rejected for the presence of visible haemolysis after centrifugation. Samples rejected for insufficient volume accounted to $1.9 \%$. $0.3 \%$ of samples were rejected for having gross lipaemia. 800 samples were rejected for having wrong/incomplete patient information, which accounted for rejection of $0.8 \%$.

\section{CONCLUSION}

The overall rate of rejection of samples is $8.52 \%$, which is very high. Haemolysis was major reason for rejection of samples collected from OPD and error due to insufficient sample volume was found to be equal in both type of samples collected from OPD and indoor patients.

\section{KEYWORDS}

Pre-analytical Errors, Hemolysis, Lipemia.

HOW TO CITE THIS ARTICLE: Barapatre AR, Jadhao AN, Lokhande MC. Evaluation of types of pre-analytical errors in clinical chemistry laboratory. J. Evolution Med. Dent. Sci. 2016;5(66):4722-4725, DOI: 10.14260/jemds/2016/1076

\section{INTRODUCTION}

Biochemistry laboratories provide many complex services such as diagnostic testing, clinical services, and sample collection. The main aim for the diagnostic service is to get correct result on correct patient to the requesting doctor without unnecessary delays. It is also the duty of laboratory to protect patients from wrong or potentially wrong results.

Typical request test report cycle consists of three phases, pre-analytical, analytical, and post-analytical phases.

Errors arising during the sample processing are classified into pre-analytical, analytical, and post-analytical errors depending upon their source and time of presentation respectively. ${ }^{1}$ Overall, the three processes combined are

Financial or Other, Competing Interest: None.

Submission 20-06-2016, Peer Review 04-08-2016,

Acceptance 11-08-2016, Published 17-08-2016.

Corresponding Author:

Dr. Amit Ramesh Barapatre,

Room No. 415,

Department of Biochemistry,

Fourth Floor, College Building,

TNMC NAIR and B.Y.L. Ch. Hospital,

Mumbai Central-08.

E-mail: amit12patre@gmail.com

DOI: $10.14260 /$ jemds/2016/1076 called as 'total testing processes.' Errors can arise at any step and lead to a faulty report generation that can affect patient care like misdiagnosis, improper treatment, mismatched blood transfusion, and so on can be sometimes be fatal.

Pre-analytical error is anything that occurs before a sample is analysed that may compromise the accuracy or integrity of the result. The pre-analytical phase of the total laboratory testing process is where the majority of laboratory errors occur. Pre-analytical errors can occur at the time of patient assessment, test order entry, request completion, patient identification, specimen collection, specimen transport, or specimen receipt in the laboratory. A report by Bonini and colleagues found that pre-analytical errors predominated in the laboratory ranging from $31.6 \%$ to $75 \%{ }^{2}$

\section{There are Two Main Types of Pre-Analytical Errors: ${ }^{3}$}

1. Identification Problems.

2. Sample Problem.

Correct identification of a sample is very important as it is the link between the patient and the tests the doctor has requested to be performed. There are 5 main identification problems as defined by RCPA KIMMS (Key Incident Management and Monitoring System) Quality Assurance Program. 


\section{Unlabelled Sample}

Mislabelled sample-any mismatch or discrepancy of identification.

Insufficiently labelled sample-less than two identifiers.

Transfusion labelling requirements-no collector signature, no time, and date of collection.

Sample suspected to be from wrong patient-wrong blood in tube.

A sample problem is when the sample supplied for testing is not suitable to be used for the tests requested. If these samples were analysed the test result reported will not give an accurate representation of the patient's condition. This may mislead the doctor when interpreting the result for diagnosis or treatment. The laboratories are very good at detecting these problems during the analytical phase. These samples are rejected and repeat samples are requested. These sample problems are due to collection-related issues. Mostly, they are due to inadequate collection technique and knowledge of appropriate procedures. In some cases, the clinical condition of the patient can also cause these sample problems. These samples are not suitable to be analysed, so they must be rejected and a repeat sample requested.

- There are several reasons why a sample may not be suitable for testing as defined by KIMMS (Key Incident Monitoring and Management System) Quality Assurance Program.

- Sample not collected.

- Incorrect sample type.

- Haemolysed sample.

- Clotted sample.

- Incorrect fill level of sample - e.g. coagulation tests.

- Insufficient sample.

- Contaminated sample - e.g. sample taken from drip arm.

- Incorrect sample storage or transport.

Pre-analytical and post-analytical phases of sample processing accounts for $93 \%$ of errors. ${ }^{4}$ The pre-analytical errors causes burden on laboratory and put a credibility of laboratories at stake.

The goal of present research is to analyse incidence of different pre-analytical errors and steps taken to prevent them during the sample processing in clinical Biochemistry Department during the three years period.

\section{MATERIALS AND METHODS}

Government Medical College and Hospital Nagpur is super speciality centre in Nagpur. It is 1200-bedded hospital offering specialised medical treatment to about $3,50,000$ patients in OPD and 25,000 patients in general and private wards every year.

GMCH Nagpur has a biochemistry laboratory equipped with state of art Randox auto analyser and other facilities for sample collection and processing. Blood specimens from the OPD are collected by laboratory personnel at centralised collection centre. Samples from wards are collected by doctors only and then delivered to staff by paramedical staff.

Total of 1,22,785 samples were collected from outpatient department and patients admitted in wards during period of one year from June 2010-July 2011. Out of these samples, 60,244 samples were collected from OPD patients and 62,541 samples were collected from indoor patients. Samples are collected using the Vacutainers.
Paramedical staff and laboratory personnel are trained thoroughly and routinely for sample collection. Upon receiving the sample, lab supervisor checks the sample and if errors are found then entries are made in logbook. The data generated is reviewed on the monthly basis.

The venous blood samples are collected unsuitable according to following criteria inappropriate volume, haemolysed and lipaemic samples, missing/wrong patient identification. The number of samples reported under lipaemia on our study reports is actually a measure of the turbidity of the sample. Index values are obtained by adding increasing amounts of an emulsion of fat to a sample (Intralipid $®$ ) and assessing the effect the emulsion has on chemistry values. In reality, this correlates weakly to the triglyceride concentration in the sample.

In our study, all the samples, which were rejected for lipaemia were markedly turbid. All the samples, which were rejected for the haemolysis were classified according to haemolytic index and all samples appeared red to markedly red in colour.

\section{RESULTS}

Out of 62,541 inpatient samples collected and screened over a period, pre-analytical errors were observed in 4,611 samples, which is approximately $3.75 \%$ of the total blood samples received. The different types of errors were also screened and their distribution was calculated. Majority of the rejected samples were of insufficient volume. Insufficient volume of samples accounted for rejection of 2428 samples, which is $1.9 \%$ of total samples received during this period.

Haemolysis was another important reason for rejection of 1360 samples, which accounts for $1.1 \%$ of total samples received during the study period.

500 samples were rejected for the gross lipaemia and it accounted for rejection of $0.4 \%$ of total samples received during this period.

323 samples were rejected for the wrong/incomplete patient information (wrong/absent slips, absent registration number). It accounted for rejection of $0.26 \%$ of total samples received during this period.

Similarly, the samples collected from the OPD were screened for the presence of pre-analytical errors and distribution of different types of errors was calculated. Out of 60,244 samples collected, total numbers of 5,552 samples were rejected for the presence of pre-analytical errors. This accounted for rejection of $4.5 \%$ of total samples received during this period.

Out of total 5,552 samples rejected, 3,329 samples were rejected for the visible haemolysis after centrifugation. This accounted for $2.7 \%$ of total blood samples collected during this period.

Another important reason for rejection of samples collected from OPD was insufficient sample volume. Total of 1200 samples were rejected for insufficient sample volume and this accounted for $1.9 \%$ of total samples received during this period.

223 samples were rejected because of the presence of gross lipaemia. This accounted for $0.3 \%$ of total samples received during period.

800 samples were rejected for the wrong/absent patient information and this constitutes $0.8 \%$ of the total samples received during this period. 


\begin{tabular}{|c|c|}
\hline \multicolumn{2}{|c|}{ Guide to Lipaemia Index } \\
\hline Lipaemia Index & Gross Appearance \\
\hline$<30$ & No turbidity \\
\hline $30-60$ & Slightly turbid (hazy) \\
\hline $60-120$ & Moderately turbid (milky) \\
\hline$>120$ & Markedly turbid (creamy) \\
\hline \multicolumn{2}{|c}{ Table 1 } \\
\hline
\end{tabular}

\begin{tabular}{|c|c|c|}
\hline HI & Appearance of Serum & Degree of Haemolysis \\
\hline$<20$ & Clear & No Haemolysis \\
\hline $20-100$ & Pink Tinged & Mild Haemolysis \\
\hline $100-300$ & Red & Moderate haemolysis \\
\hline$>300$ & Dark Red & Severe haemolysis \\
\hline \multicolumn{3}{|c|}{ Table 2 } \\
\hline
\end{tabular}

\begin{tabular}{|c|c|c|c|c|c|c|}
\hline & OPD & \% & IPD & \% & OPD+IPD & Total \% \\
\hline Total samples & 60,244 & & 62,541 & & $1,22,785$ & \\
\hline Misidentification & 800 & $0.8 \%$ & 323 & $0.26 \%$ & 1,123 & $1.6 \%$ \\
\hline Insufficient volume & 1,200 & $1.9 \%$ & 2,428 & $1.9 \%$ & 3,628 & $3.8 \%$ \\
\hline Clotted samples & 42 & 0.0023 & 56 & $0.003 \%$ & 98 & $0.0053 \%$ \\
\hline Incorrect vials & 21 & $0.0019 \%$ & 34 & $0.0021 \%$ & 55 & $0.004 \%$ \\
\hline Lipaemic & 223 & $0.3 \%$ & 500 & $0.4 \%$ & 723 & $0.7 \%$ \\
\hline Haemolysed & 3,329 & $2.7 \%$ & 1,360 & $1.1 \%$ & 4,689 & $3.8 \%$ \\
\hline \multicolumn{7}{|c|}{ Table 3 } \\
\hline
\end{tabular}

Table 1 show the lipaemia index marker, which was used as guide to select the lipaemic samples for our study and Table 2 shows the haemolysis index used to select haemolytic samples for our study. Table 3 shows the total number of indoor samples and outdoor samples collected over period of study and the distribution of pre-analytical errors during study period.

\section{DISCUSSION}

Remarkable advances in instrument technology, automation, and computer science have greatly simplified many aspects of previously tedious tasks in laboratory diagnostics, creating greater volume of routine work, and significantly improving quality of results of laboratory testing.

Results have shown that pre-analytical phase of sample processing accounts for more than $75 \%$ of the laboratory errors. $^{5}$ It is estimated that $90 \%$ of clinical decisions are based on information derived from the laboratory results, so ensuring optimal quality is very crucial to the patients. ${ }^{6}$ The role of laboratory is to provide quality service and achieving this goal is of paramount importance.

Currently, the pre-analytical errors accounts up to $93 \%$ of all errors made in laboratory diagnostics. Majority of these errors arises from problems in patient preparation, sample collection, transportation, storage, and preparation for analysis.

In our research, we have studied the pre-analytical errors in two different categories of samples, samples from indoor patients, and sample collected from OPD.

Out of 60,244 samples collected from OPD, pre-analytical errors were found to be present in 5,552 samples. The frequency of pre-analytical errors was found to be $4.5 \%$ of total samples received during this period. This error is large considering the total samples collected from OPD and the fact that most of clinical decisions depend upon laboratory results.

Similarly out of 62,541 inpatient samples collected and screened over a period, pre-analytical errors were observed in 4,611 samples, which is approximately $3.75 \%$ of the total blood samples received.

Thus, total amount of pre-analytical error was found to be close to $8 \%$. The amount of pre-analytical error was found to be slightly less in indoor patients sample collection as compared to OPD patients. There has been varied information on error rate within whole lab testing procedure (0.1-9.3\%). ${ }^{7}$

Our study is in accordance with Pleboni and Carroro who in their research also observed that majority of errors in laboratory results due to the problem in pre-analytical phase.

In our study, visible haemolysis accounted for rejection of majority of the samples collected from OPD. It accounted for rejection of $2.7 \%$ of total samples collected over a period of study. Similarly, haemolysis was responsible for rejection of 1,360 samples from indoor patients, which accounted for $1.1 \%$ of total samples. Thus, haemolysis accounted for $3.8 \%$ of total pre-analytical error during period of study. The percentage of haemolysis was found to be more in samples collected from OPD as compared to indoor patient samples. One logical explanation for this is very large patient load and fewer workforces in the OPD hours.

There are number of reasons, which cause haemolysis during the sample collection. An improper choice in the venepuncture site such as drawing from a distal site to the antecubital region of the arm rather than drawing from an antecubital site has been shown to result in more haemolysis. Prolonged tourniquet time causes the interstitial fluid to leak into the tissue and cause haemolysis. Cleansing the venepuncture site with alcohol and not allowing the site to dry may cause haemolysis. The use of a small-bore needle resulting in a large vacuum force applied to the blood may cause shear stress on the red blood cells causing them to rupture. The use of a large bore needle may result in a much faster and more forceful flow of blood through the needle resulting in haemolysis.

In one study by Jay and colleagues, majority of haemolysed samples could be attributed to in vitro processes resulting from incorrect sampling procedure and transportation. ${ }^{8}$

In spite of above reasons, haemolysis is easily preventable cause of pre-analytical error. As a part of our efforts to achieve goal of high quality laboratory tests, we carry out routine training of our laboratory staff and proper standard operating procedures have also been developed to facilitate laboratory personnel to adopt ideal phlebotomy practices.

Another important reason for rejection of large number of samples from both OPD and indoor patients is the inappropriate sample volume. Inappropriate sample volume was responsible for rejection of 2428 (1.9\%) and 1200 samples $(1.95 \%)$ from indoor patients and OPD patients 
respectively. Thus, total amount of error due to inappropriate volume of sample was around 3.8\%. This error percentage was found to be equal to the percentage of samples rejected for haemolysis. The main reason for inappropriate sample volume were difficult sampling as in paediatric patients and patients suffering from chronic debilitating diseases in whom thin veins are difficult to localise. In our study, rejections due to inappropriate volume of sample was found to be slightly more in indoor patients as compared to OPD samples. This may be due to repeated sampling from patients who are admitted to ward for longer period of time for treatment of chronic diseases or repeated sampling in less volume of time.

Repeated drawing of samples from accessible sites cause collapsing and thinning of veins resulting in less volume of sample. In OPD patient, inappropriate sample of volume may result due to large patient load. Main reasons for inappropriate sample volume in OPD patient can be due to very high patient load, less phlebotomists, along with patient's noncompliance, and difficult sampling. In addition to this sample, collection from OPD is carried out during the fixed hours only. All this factors contributed to inappropriate sample volume.

In our study, we found that 500 samples from samples collected from OPD were rejected for having gross lipaemia. This accounted for rejection of $0.4 \%$ of total samples collected over period of study. Similarly, 223 samples from indoor patients were rejected for having gross lipaemia. This accounted for rejection of $0.1 \%$ of total samples collected over a period of study. Thus, total amount of error of samples rejected due to gross lipaemia was found to be $0.5 \%$. In our study, we found that samples rejected due to lipaemia was found to be more in OPD patients than the samples collected from indoor patients. The reason behind this when traced was found to be inadequate preparation of patients. Most of patients when enquired about the instructions given to them for blood collection had said that they were asked to be in fasting state and they were properly explained the meaning of fasting state. Most of the patients wrongly believed that their preparation for fasting state was adequate believed that the exact time that has to pass since the last meal does not matter. In the hospital setting, a certain proportion of lipaemic samples can't be avoided since patients are admitted to the emergency services in various times of the day and various intervals since their last meal. This contributed to the lipaemic error occurring in samples collected from indoor patients.

In our study, we found that 323 samples from samples collected from indoor patients were rejected for wrong/incomplete patient information (i.e., wrong requisition slip, without requisition slip, central registration number, ward not mentioned). This accounts for rejection of $0.26 \%$ of total samples collected over a period of study. Similarly, it was found that 800 samples from OPD were rejected for wrong/incomplete patient information. This accounts for rejection of $0.8 \%$ of total samples collected over a period of study. Thus, total amount of error due to wrong/incomplete patient information is almost $1 \%$. It has been observed that the clinicians often send incomplete slips with the samples. This could be due to excessive patient load or lack of awareness regarding patient information. Some of information missing from samples collected from indoor patients could be retrieved back due to painstaking efforts of our laboratory personnel. Same cannot be followed for the OPD patients.

\section{CONCLUSION}

The role of clinical laboratories in diagnostic medicine has been very well established. Radical changes have occurred in organisation, number and types of tests, and role of medical laboratories in healthcare over recent years. The affirmation of a new role for laboratory professionals calls for greater accuracy and a more stringent test selection and interpretation of results. Patient safety emphasises the reporting, analysis, and prevention of medical errors that often lead to adverse events. Besides carrying serious harms to patient health, medical errors translate into a huge amount of money wiped out of the national and international economy.

The increasing awareness of the issues involving medical errors has cast a spotlight on factors that contribute to the resulting adverse events and has also made the clinical laboratories subject of scrutiny as essential part of the overall healthcare system. Automation, computers have greatly simplified the many aspects of previously tedious tasks creating the greater volume of routine work as well as significantly improving the analytical rate over time. Therefore, mistake outside the analytical phase of testing seems more likely to affect the usefulness of laboratory results in patient care.

The practice of keeping a record of errors at all stages of analysis and then devising corrective strategies for their prevention can gradually free a laboratory from such errors. Reducing pre-analytical errors not only optimises patient safety and care, but also improves efficiency of Biochemistry Laboratory service by reducing rework.

To conclude, it is important to continually educate internal and external sample collectors to practice ideal phlebotomy and sample transportation procedures and to make them understand consequences of pre-analytical errors. We as biochemist should work in conjunction with clinicians for the patient safeguard. Adaptation of quality control in all phases and not merely the analytical processes is necessary to safeguard patient interests and to deliver our services to society.

\section{REFERENCES}

1. Wallin O, Soderberg J, Guelpen VB. Pre-analytical venous blood sampling practices demand improvement-a survey of test-request management, test-tube labelling, and information search procedures. Clin Chim Acta 2008;391(1-2):91-7.

2. Bonini P, Plebani M, Ceriotti F, et al. Errors in laboratory medicine. Clin Chem 2002;48(5):691-8.

3. Lundberg GD. Acting on significant laboratory results. JAMA 1981;245(17):1762-3.

4. Boone DJ. Governmental perspectives on evaluating laboratory performance. Clin Chem 1993;39(7):1461-5, discussion 1466-7.

5. Plebani M, Carraro P. Mistakes in a stat laboratory: types and frequency. Clin Chemistry 1997;43(8 Pt 1):1348-51.

6. Toybert ME, Chevret S, Forsman RW, et al. Why is the laboratory an afterthought for managed care organisations? Clin Chem 1996;42(5):813-6.

7. Szecsi PB, Odum L. Error tracking in clinical biochemistry laboratory. Clin Chem Lab Med 2009;47(10):1253-7.

8. Jay DW, Provasek D. Characterisation and mathematical correction of haemolysis interference in selected Hitachi 717 assays. Clin Chem 1993;39(9):1804-10. 